\title{
Detection or Modulation at 35 Gbit/s with a Standard CMOS-processed Optical Waveguide
}

\author{
Dietmar Korn ${ }^{1}$, Hui Yu ${ }^{2}$, David Hillerkuss ${ }^{1}$, Luca Alloatti ${ }^{1}$, Christoph Mattern ${ }^{1}$, Katarzyna Komorowska ${ }^{2}$, \\ Wim Bogaerts ${ }^{2}$, Roel Baets ${ }^{2}$, Joris Van Campenhout ${ }^{3}$, Peter Verheyen ${ }^{3}$, Johan Wouters ${ }^{3}$, Myriam Moelants ${ }^{3}$, \\ Philippe Absil ${ }^{3}$, Christian Koos ${ }^{1}$, Wolfgang Freude ${ }^{1}$, Juerg Leuthold ${ }^{1}$ \\ ${ }^{1}$ Institute of Photonics and Quantum Electronics (IPQ) and Institute of Microstructure Technology (IMT), \\ Karlsruhe Institute of Technology(KIT), 76131 Karlsruhe, Germany \\ korn@kit.edu,Juerg.Leuthold@kit.edu \\ ${ }^{2}$ Department of Information Technology, Ghent University-imec, Center for Nano- and Biophotonics (NB Photonics), \\ St.-Pietersnieuwstraat 41, 9000 Ghent, Belgium \\ ${ }^{3}$ imec, Kapeldreef 75, 3001 Leuven, Belgium
}

\begin{abstract}
Light modulation and detection within a single SOI waveguide is demonstrated at $1550 \mathrm{~nm}$. Multi-functional devices allow simplified transceiver systems. Savings in the number of fabrication steps increase the yield and reduce costs.

OCIS codes: Integrated optics: 130.4110 Modulators, Detectors: 040.6040 Silicon, Integrated optics: 130.3060 Infrared.
\end{abstract}

\section{Introduction}

Waveguide-based photodetectors and modulators are key components for highly integrated photonic circuits. Silicon photonics is particularly appealing, because of the prospect to produce chips using existing CMOS infrastructure. Modulators [1] usually rely on free-carrier dispersion in all-silicon structures, whereas detectors either exploit defect states [2] or require deposition of Germanium [3] on silicon substrates. Combining both functions on the same chip requires substantial processing effort and results in a decrease of yield.

In this paper, we demonstrate for the first time that a single device can be used to modulate and to detect broadband communication signals with data rates of $35 \mathrm{Gbit} / \mathrm{s}$. The device was fabricated using only the most common and reliable CMOS processes: Silicon dry etching and ion implantation. This significantly reduces the number of processing steps and allows for less complex fabrication runs with lower risk.

\section{Device design and fabrication}

The layout of our phase modulator is shown in Fig. 1. The modulator exploits carrier depletion in a $3 \mathrm{~mm}$ long ridge waveguide which was doped symmetrically with $\mathrm{p}$ and $\mathrm{n}$ dopants (concentrations of boron and phosphorous both $2 \times 10^{18} \mathrm{~cm}^{-3}$ ) and annealed at $1075{ }^{\circ} \mathrm{C}$ for $10 \mathrm{~s}$. Waveguides are defined on a standard p-doped SOI wafer (concentration $1 \times 10^{15} \mathrm{~cm}^{-3}$ ) with $2 \mu \mathrm{m}$ buried oxide layer. Processing was performed in a standard CMOS line with $193 \mathrm{~nm}$ optical lithography. We start with a $220 \mathrm{~nm}$ high Si layer, use $70 \mathrm{~nm}$ dry-etching to define the ridge waveguides (500 nm width) and shallow-etching for the grating couplers. Strip waveguides with a width of $500 \mathrm{~nm}$ are then fabricated by fully etching the device layer around the phase modulation sections. Pt/Au coplanar waveguide electrodes were realized in a ground-signal-ground configuration (widths: signal $6 \mu \mathrm{m}$, ground ca. $100 \mu \mathrm{m}$, gap 3.5 $\mu \mathrm{m}$; pads larger but not shown). The electrodes are deposited on a BCB layer to enable crossings of optical waveguides, and are in contact only with strongly doped (silicide) $\mathrm{p}++$ and $\mathrm{n}++$ regions. These are connected by $400 \mathrm{~nm}$ wide and $1 \mu \mathrm{m}$ long arms to the p-n-doped ridge waveguide. The doping was optimized for modulator applications.

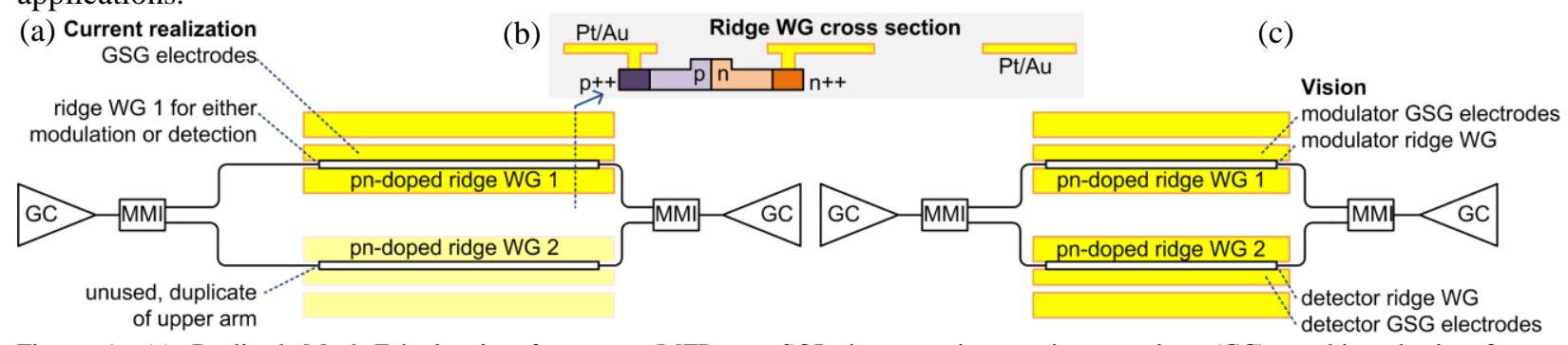

Figure 1. (a) Realized Mach-Zehnder interferometer (MZI) on SOI, incorporating grating couplers (GC), multi-mode interference couplers (MMI), and carrier-depleted ridge waveguides. (b) The structure's cross-section. WG 1 and WG 2 are identical; WG 1 was used for detection and modulation experiments. (c) Vision: Use WG 1 for modulation, WG 2 for detection. Use case 1: Half-duplex transceiver, i.e. switch between modulation or detection mode. Use case 2: Pulse carving without the need for an external trigger; WG 2 is used to monitor the incoming signal to synchronize an on-chip clock circuit to the signal. An electronic driver circuit then feeds WG 1.

The operation principle of detection of photons with sub-bandgap energies in Si has been shown and explained by Knights et al. [4], relying on $\mathrm{Si}^{+}$impurities to introduce defects associated with sub-bandgap states and (p, n)- 
dopants (phosphorus, boron). Detection without $\mathrm{Si}^{+}$impurities showed weaker performance [5]. We use the same waveguide designed for phase modulation to detect light, suspecting that incomplete annealing of ( $\mathrm{p}, \mathrm{n})$ implantation will leave crystal defects. In this first demonstration the focus had been on optimizing the quality of the modulator operation. In the future, when we are able to co-integrate more elaborate designs and electronics on the same chip, the detector performances will be optimized (e. g., the dark current could be decreased and the responsivity increased).

An imbalanced (arm length difference $40 \mu \mathrm{m}$ ) Mach-Zehnder interferometer (MZI) with multi-mode interference couplers (MMIs) was connected to fibers via grating couplers (GCs, coupling loss $5 \mathrm{~dB}$ ), see Fig. 1. This amplitude modulator works over a wide wavelength range. In the following, we use only one arm to show both modulation and detection, because the design allotted insufficient space to contact both coplanar electrical waveguides at the same time. However, more functionality can be envisioned for this device: Imagine arm 1 of the MZI connected to driver electronics for modulation, but arm 2 permanently attached to the receiver electronics on-chip. While such a device can act as a half-duplex transceiver, also signal monitoring and feedback for immediate signal processing are conceivable. Operating WG 2 at the same bias as WG 1 gives the best modulation extinction ratio, while a higher bias on WG 2 could be temporally used to increase receiver bandwidth and analyze the incoming signal.

\section{Modulator and detector performance}

We operate arm 1 at $5.7 \mathrm{~V}$ reverse bias and drive it with a non-return-to-zero (NRZ) pseudo random bit sequence (PRBS) of length $2^{7}-1$ resulting an optical on-off-keying (OOK) signal. The data stream is analyzed with a preamplified receiver, a digital communication analyzer (DCA) and and a bit error ratio tester (BERT), and it produces open eye diagrams as shown in Fig. 2a. This proves error-free operation $\left(\mathrm{BER}<10^{-10}\right)$ at $35 \mathrm{Gbit} / \mathrm{s}$.

The detector's reverse bias is set to $7.3 \mathrm{~V}$, close to the breakdown voltage, and causes a dark current of $34 \mu \mathrm{A}$. Varying the optical power near $14 \mathrm{dBm}$ in WG 1 results in a responsivity of $0.03 \mathrm{~A} / \mathrm{W}$. Using a signal created with a lithium niobate (LN) modulator at $35 \mathrm{Gbit} / \mathrm{s}$, which is launched with a power of $14 \mathrm{dBm}$ into WG1, a BER below $10^{-6}$ is measured, well below the threshold for second-generation forward-error correction (FEC), see Fig. $2 \mathrm{~b}$. Measurements of reverse bias current vs. optical input power (not shown here) exclude two-photon absorption as a physical effect to explain detection at photon energies smaller than the bandgap.
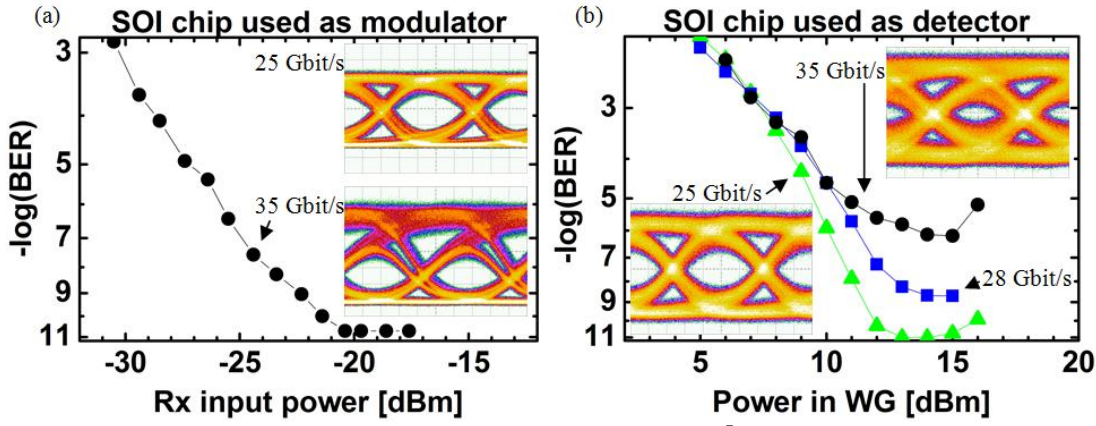

Figure 2 Performance characterization around $1550 \mathrm{~nm}$ by data transmission of a PRBS $\left(2^{7}-1\right)$ using NRZ OOK. (a) BER and open eye diagrams for modulation with SOI chip (reverse biased at $5.7 \mathrm{~V}$ ) using commercial receiver. (b) BER and open eye diagrams for detection with SOI chip (reverse biased at $7.1 \mathrm{~V}$ ) of optical signal (generated with LN modulator) using electronic amplifier.

4. Conclusion and acknowledgements

High speed modulation or detection in the same SOI waveguide from fabrication relying only on dry etching and ion implantation has been demonstrated for the first time, allowing for multiple-purpose devices, e.g. instant signal processing with less effort. The striking extend to which silicon can be used for sub-bandgap light detection without dedicated fabrication steps is revealed, which allows for saving costs but also implicates for other detection concepts on SOI (e.g. plasmonics) to consider the influence on responsivity from silicon itself.

This work was supported by the EU-FP7 project SOFI (grant 248609), the BMBF project MISTRAL, and by CFN and KSOP in Karlsruhe. We acknowledge the efforts of IMEC's 200-mm pline in device fabrication and IMEC's Core Partner Program.

[1] G. T. Reed, G. Mashanovich, F. Y. Gardes, D. J. Thomson, "Silicon optical modulators", Nature Photon. 4, 518-526 (2010).

[2] M. W. Geis et al. , "Silicon waveguide infrared photodiodes with $>35 \mathrm{GHz}$ bandwidth and phototransistors with $50 \mathrm{~A} / \mathrm{W}$ response", Optics Express, 17, 5193-5204 (2009).

[3] J. Michel, J. Liu, L. C. Kimerling, "High-performance Ge-on-Si photodetectors“, Nature Photon. 4, 527 - 534 (2010)

[4] D. F. Logan, P. E. Jessop, A. P. Knights, "Modeling Defect Enhanced Detection at $1550 \mathrm{~nm}$ in Integrated Silicon Waveguide Photodetectors", Journal of Lightwave Technology, Vol. 27, Issue 7, pp. 930-937 (2009)

[5] Y. Zhao et al., "Photocurrent Effect in Reverse-Biased p-n Silicon Waveguides in Communication Bands", Chin. Phys. Lett. 28, 074216 (2011) 